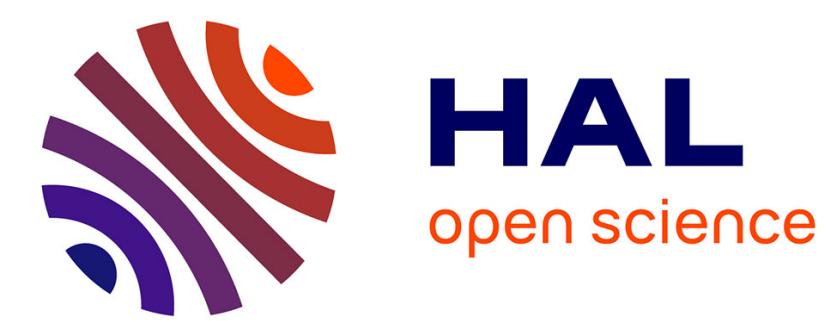

\title{
Linear and nonlinear response of the Vlasov system with nonintegrable Hamiltonian \\ Shun Ogawa
}

\section{To cite this version:}

Shun Ogawa. Linear and nonlinear response of the Vlasov system with nonintegrable Hamiltonian. Physical Review E , 2017, 96 (1), pp.012112 10.1103/PhysRevE.96.012112 . hal-01662966

\section{HAL Id: hal-01662966 https://hal.science/hal-01662966}

Submitted on 18 May 2018

HAL is a multi-disciplinary open access archive for the deposit and dissemination of scientific research documents, whether they are published or not. The documents may come from teaching and research institutions in France or abroad, or from public or private research centers.
L'archive ouverte pluridisciplinaire HAL, est destinée au dépôt et à la diffusion de documents scientifiques de niveau recherche, publiés ou non, émanant des établissements d'enseignement et de recherche français ou étrangers, des laboratoires publics ou privés. 


\title{
Linear and nonlinear response of Vlasov system with non-integrable Hamiltonian
}

\author{
Shun Ogawa ${ }^{1, *}$ \\ ${ }^{1}$ Aix Marseille Univ., Université de Toulon, CNRS, CPT, Marseille, France
}

\begin{abstract}
Linear and nonlinear response formulae taking into account all Casimir invariants are derived without use of angle-action variables of a single particle (mean-field) Hamiltonian. This article deals mainly with the Vlasov system in a spatially inhomogeneous quasi-stationary state whose associating single particle Hamiltonian is not integrable and has only one integral of the motion, the Hamiltonian itself. The basic strategy is to restrict the form of perturbation so that it keeps Casimir invariants within a linear order, and the single particle's probabilistic density function is smooth with respect to the single particle's Hamiltonian. The theory is applied for a spatially two dimensional system and is confirmed by numerical simulations. A nonlinear response formula is also derived in the similar manner.
\end{abstract}

\section{INTRODUCTION}

Long range interaction systems show several phenomena which are out of scope of the equilibrium statistical mechanics $[1,2]$. One of them is that such a system is often trapped in out-of-equilibrium quasi-stationary states (QSSs) whose duration gets to be longer as the number $N$ of elements in a system and diverges when the large population limit $N \rightarrow \infty$ is taken [1-5]. Then, if the system of interest is huge enough, the relaxation time is so long that one cannot see the thermal equilibrium state. It is hence interesting to investigate the non-equilibrium statistical mechanics or thermodynamics of QSSs. In particular, the topic of this article is effect of external forces in the QSSs.

When $N$ is huge enough, temporal evolution of the longrange interaction system is well described by the Vlasov equation [6-8] (also called the collisionless Boltzmann equation [3]) which describes evolution of a density function defined on a $\mu$-space, a single particle phase space. The QSSs are interpreted as stable stationary solutions to the Vlasov equation [4]. The Vlasov equation has unique solutions for each given initial state [7, 8], and thus the QSSs depend not only on macroscopic variables such as temperature and energy but also on mesoscopic things, details of the single particle density function. Thus the study on responses to external forces in QSSs should be based on the Vlasov equation.

The linear response theory for the Vlasov systems has been developed for stability analysis in self-gravitating systems [3], for looking into plasmas responses in magnetically confined plasmas [9], for computing time-asymptotic response to the external forces of long-range interaction systems in both spatially homogeneous [10] and inhomogeneous [11] QSSs and of a fluid systems [12]. By use of this theory, critical phenomena in QSSs [13] are investigated, and some informations of unforced systems are extracted by observing responses to oscillating external forces [11]. Further, the nonlinear response theory has been developed

* shun.ogawa@riken.jp, Author's current address: RIKEN BST, 2-1 Hirosawa, Wako, 351-0198Japan to investigate the response to the finite size external forces in QSSs near or on the critical point in which the linear response theory does not work [14, 15]. These response formulae have been derived only when the single particle effective Hamiltonian is integrable and the angle-action variables are used for solving test particle dynamics.

Analyzing the linearized equation with the nonintegrable effective Hamiltonian is practically important, because systems in multi-dimensional spaces are more realistic (for example, self-gravitating systems in the three dimensional (3D) space, magnetically confined hot plasmas) and their effective Hamiltonians are non-integrable in general. To tackle this problem, one method to take into account constraints a posteriori is proposed to obtain linear response formulae approximately [16]. This method provides canonical (taking into account the normalization) and micro-canonical (taking into account the normalization and the energy concervation) linear responses and other kinds of linear responses with a finite number of constraints systematically. However one cannot obtain isolated one with this method because it is practically impossible to take into account infinitely many Casimirs constraints with this method. The same problem lies in the stability analysis of the Vlasov equation [17]. If the effective Hamiltonian is not integrable, it was impossible to obtain the precise stability criterion with a finite time step procedure in general, since one should obtain an infinite number of Lagrangian multipliers associated with the Casimir's constraints [18].

The above problems in the linearized equation should be solved as the first step to understand the dynamics around the QSSs with non-integrable effective Hamiltonian. After that, we will be able to continue tackling more difficult problems on nonlinear Landau damping, nonlinear stability, nonlinear response, critical phenomena and their universality, and finite $N$ effects.

In this article, we firstly obtain the linear response of the Vlasov system in the multi-dimensional space without solving the linearized Vlasov equation and without using the angle-action variables. Let an initial state without external field be $f_{0}(\boldsymbol{q}, \boldsymbol{p})$ and a final state $f_{h}(\boldsymbol{q}, \boldsymbol{p})$ after exerting external field $h$. The linear response is obtained by restricting the form of accessible perturbation by assuming smoothness of $f_{h}$ with respect to $h$, and by taking into account the 
constraint conditions that the perturbation should be on a tangent "plane" of a constraint surface at $f_{0}$.

Further, we shall mention the nonlinear response formula [14] derived via the transient (T-) linearization method developed by Lecellotti and Dorning [19-21] to analyse the plasmas oscillation and nonlinear Landau damping. In this theory, the Vlasov equation is linearized around an "unknown" asymptotic stationary state. Solving this equation, we obtain a self-consistent equation determining the asymptotic state. The asymptotic solution is obtained by redistributing the initial density function along iso-asymptotic effective Hamiltonian sets, and this formula is called rearrangement formula [22]. The same formula is also derived for predicting the QSSs in a 1D system [23] and a 3D self-gravitating system [24] via the another consideration when non-stationary initial states satisfy a (generalized) viral condition and there is no parametric resonance. It is derived the rearrangement formula keeps Casimir invariants at the order of T-linearization. Then, the nonlinear response formula is derived in a similar manner to derive the linear response formula in this article.

This article is organized as follows: The model and the dynamics in a mean-field limit $N \rightarrow \infty$ are firstly introduced in Sec. II, and the explicit form of constraint condition coming from Casimir invariants is derived in Sec. III. Based on this constraint condition, the linear response formula is derived in Sec. IV and several examples are exhibited in Sec. V. We derive the nonlinear response formula and make a brief comment on problems in the T-linearized Vlasov equation for spatially multi-dimensional systems in Sec. VI, and summarize this article in Sec. VII.

\section{MODEL AND ITS DYNAMICS}

\section{A. Model and Vlasov equation}

Let us consider a system with long-range interaction whose Hamiltonian is

$$
H_{N}=\sum_{i=1}^{N} \frac{\left\|\boldsymbol{p}_{i}\right\|^{2}}{2}+\frac{1}{2 N} \sum_{i, j=1}^{N} V\left(\boldsymbol{q}_{i}-\boldsymbol{q}_{j}\right)+h(t) \sum_{i=1}^{N} \Phi\left(\boldsymbol{q}_{i}\right),
$$

where $\boldsymbol{q}_{i}$ denotes configuration of the $i$-th particle, $\boldsymbol{p}_{i}$ its conjugate momentum, $V$ the inter-particles (-cites) potential, and $h(t) \Phi\left(\boldsymbol{q}_{i}\right)$ interaction between the external field $h$ and the $i$-the particle. Taking the mean-field limit $N \rightarrow$ $\infty$ [6-8], the temporal evolution of this system can be described in terms of the single-particle density function $f(\boldsymbol{q}, \boldsymbol{p}, t)$ which is a solution to the Vlasov equation,

$$
\frac{\partial f}{\partial t}+\{\mathscr{H}[f], f\}=0
$$

where $\mathscr{H}[f]$ is an effective single-particle Hamiltonian,

$$
\mathscr{H}[f]=\frac{\|\boldsymbol{p}\|^{2}}{2}+\mathcal{V}[f](\boldsymbol{q})+h(t) \Phi(\boldsymbol{q}),
$$

and $\{a, b\}$ is the Poisson bracket given by

$$
\{a, b\}=\frac{\partial a}{\partial \boldsymbol{p}} \cdot \frac{\partial b}{\partial \boldsymbol{q}}-\frac{\partial a}{\partial \boldsymbol{q}} \cdot \frac{\partial b}{\partial \boldsymbol{p}} .
$$

The system is initially in a QSS, $f_{0}$, and the effective Hamiltonian

$$
\mathscr{H}_{0}(\boldsymbol{q}, \boldsymbol{p})=\mathscr{H}\left[f_{0}\right](\boldsymbol{q}, \boldsymbol{p})=\frac{\|\boldsymbol{p}\|^{2}}{2}+\mathscr{V}\left[f_{0}\right](\boldsymbol{q})
$$

has only one integral of motion, $\mathscr{H}_{0}$ itself. The $f_{0}$ is expressed as $f_{0}(\boldsymbol{q}, \boldsymbol{p})=F_{0}\left(\mathscr{H}_{0}(\boldsymbol{q}, \boldsymbol{p})\right)$ by use of a monotonically decreasing function $F_{0}$. This assumption is reasonable when we are interested in the asymptotic behavior of perturbations around the formally stable solutions to the Vlasov equation $[4,5,17,25]$. The formal stability is defined in terms of positive or negative definiteness of second variation of an invariant functional around $f_{0}$ which is a solution to the optimization problem:

$$
\begin{aligned}
& \text { maximizing } \mathscr{S}[f]=\iint s(f) d \boldsymbol{q} d \boldsymbol{p}, \\
& \text { subject to } \quad 1=\mathscr{N}[f]=\iint f d \boldsymbol{q} d \boldsymbol{p}, \\
& E=\mathscr{E}[f]=\iint \frac{\|\boldsymbol{p}\|^{2}}{2} f d \boldsymbol{q} d \boldsymbol{p}+\frac{1}{2} \iint \mathcal{V}[f] f d \boldsymbol{q} d \boldsymbol{p}
\end{aligned}
$$

where $s$ is a convex function. A formally stable solution is linearly stable [25]. By solving the optimization problem, we have a solution,

$$
f_{0}(\boldsymbol{q}, \boldsymbol{p})=\left(s^{\prime}\right)^{-1}\left(\beta \mathscr{H}_{0}+\alpha\right) \equiv F_{0}\left(\mathscr{H}_{0}\right),
$$

where $s^{\prime}$ denotes $d s(x) / d x$, and $\alpha$ and $\beta$ are Lagrangian multipliers with respect to the normalization and the energy conservation. Since $s$ is convex, then the inverse of its first derivative $\left(s^{\prime}\right)^{-1}$ is a strictly decreasing function. The parameter $\beta$ must be positive.

\section{B. Linear response}

The external field $h(t)$ is turned on and it converges to a constant $h(t) \rightarrow h$ as $t \rightarrow \infty$. In the previous studies [10-12] the asymptotic linear response $\delta f$ is obtained by solving the linearized Vlasov equation around $f_{0}$,

$$
\left.\frac{\partial g_{\mathrm{p}}}{\partial t}+\left\{\mathscr{H}_{0}, g_{\mathrm{p}}\right\}+\left\{\mathcal{V}\left[g_{\mathrm{p}}\right]+h(t)\right) \Phi, f_{0}\right\}=0
$$

where $g_{\mathrm{p}}(t) \sim O(h)$ is a perturbation around $f_{0}$, and by taking the limit, $\delta f=\lim _{t \rightarrow \infty} g_{\mathrm{p}}(t)$. The angle-action variables of the Hamiltonian $\mathscr{H}_{0}$ is necessary to solve the linearized Vlasov equation, but it is impossible in general for multidimensional systems. To avoid this problem, we focus on constraint conditions restricting a form of perturbations, and we obtain the linear response $\delta f$ without solving the linearized Vlasov equation. 


\section{CASIMIR INVARIANTS}

We assume that $f$ and its derivatives converge to 0 rapidly enough as $\|\boldsymbol{p}\| \rightarrow \infty$. Further, $f$ and its derivatives are assumed to vanish on the boundary of spatial domain or the system has periodic boundary condition with respect to $\boldsymbol{q}$. Under these assumptions, it is shown (see Appendix. A) that the Vlasov equation keeps values of Casimir functionals,

$$
\mathscr{C}[f]=\iint c(f(\boldsymbol{q}, \boldsymbol{p}, t)) d \boldsymbol{q} d \boldsymbol{p},
$$

for any smooth function $c$. The linearized Casimir conservation condition is expressed as that the accessible perturbation $\delta f$ satisfies

$$
\iint c^{\prime}\left(f_{0}(\boldsymbol{q}, \boldsymbol{p})\right) \delta f(\boldsymbol{q}, \boldsymbol{p}) d \boldsymbol{q} d \boldsymbol{p}=0
$$

where $c^{\prime}(x)=d c / d x$ for any smooth function $c$. Since $f_{0}(\boldsymbol{q}, \boldsymbol{p})=F_{0}\left(\mathscr{H}_{0}(\boldsymbol{q}, \boldsymbol{p})\right), F_{0}$ is a monotonically decreasing function, and $c$ is chosen arbitrarily, the constraint condition (9) is equivalent to the condition,

$$
\begin{aligned}
0 & =\iint R\left(\mathscr{H}_{0}(\boldsymbol{q}, \boldsymbol{p})\right) \delta f(\boldsymbol{q}, \boldsymbol{p}) d \boldsymbol{q} d \boldsymbol{p} \\
& =\iint R\left(\mathscr{H}_{0}(\boldsymbol{q}, \boldsymbol{p})\right)\langle\delta f\rangle_{\mathscr{H}_{0}(\boldsymbol{q}, \boldsymbol{p})} d \boldsymbol{q} d \boldsymbol{p},
\end{aligned}
$$

for any function $R$ [17]. The second equality is shown as follows:

$$
\begin{aligned}
& \iint R\left(\mathscr{H}_{0}(\boldsymbol{q}, \boldsymbol{p})\right)\langle\delta f\rangle_{\mathscr{H}_{0}(\boldsymbol{q}, \boldsymbol{p})} d \boldsymbol{q} d \boldsymbol{p}=\iint R\left(\mathscr{H}_{0}(\boldsymbol{q}, \boldsymbol{p})\right) \frac{\iint \delta f\left(\boldsymbol{q}^{\prime}, \boldsymbol{p}^{\prime}\right) \delta\left(\mathscr{H}_{0}(\boldsymbol{q}, \boldsymbol{p})-\mathscr{H}_{0}\left(\boldsymbol{q}^{\prime}, \boldsymbol{p}^{\prime}\right)\right) d \boldsymbol{q}^{\prime} d \boldsymbol{p}^{\prime}}{\iint \delta\left(\mathscr{H}_{0}(\boldsymbol{q}, \boldsymbol{p})-\mathscr{H}_{0}\left(\boldsymbol{q}^{\prime \prime}, \boldsymbol{p}^{\prime \prime}\right)\right) d \boldsymbol{q}^{\prime \prime} d \boldsymbol{p}^{\prime \prime}} d \boldsymbol{q} d \boldsymbol{p} \\
& =\iint R\left(\mathscr{H}_{0}(\boldsymbol{q}, \boldsymbol{p})\right)\left[\iint \frac{\delta f\left(\boldsymbol{q}^{\prime}, \boldsymbol{p}^{\prime}\right) \delta\left(\mathscr{H}_{0}(\boldsymbol{q}, \boldsymbol{p})-\mathscr{H}_{0}\left(\boldsymbol{q}^{\prime}, \boldsymbol{p}^{\prime}\right)\right)}{S\left(\mathscr{H}_{0}(\boldsymbol{q}, \boldsymbol{p})\right)} d \boldsymbol{q}^{\prime} d \boldsymbol{p}^{\prime}\right] d \boldsymbol{q} d \boldsymbol{p} \\
& =\iint \delta f\left(\boldsymbol{q}^{\prime}, \boldsymbol{p}^{\prime}\right)\left[\iint \frac{R\left(\mathscr{H}_{0}(\boldsymbol{q}, \boldsymbol{p})\right) \delta\left(\mathscr{H}_{0}(\boldsymbol{q}, \boldsymbol{p})-\mathscr{H}_{0}\left(\boldsymbol{q}^{\prime}, \boldsymbol{p}^{\prime}\right)\right)}{S\left(\mathscr{H}_{0}(\boldsymbol{q}, \boldsymbol{p})\right)} d \boldsymbol{q} d \boldsymbol{p}\right] d \boldsymbol{q}^{\prime} d \boldsymbol{p}^{\prime} \\
& =\iint \delta f\left(\boldsymbol{q}^{\prime}, \boldsymbol{p}^{\prime}\right) \frac{R\left(\mathscr{H}_{0}\left(\boldsymbol{q}^{\prime}, \boldsymbol{p}^{\prime}\right)\right) S\left(\mathscr{H}_{0}\left(\boldsymbol{q}^{\prime}, \boldsymbol{p}^{\prime}\right)\right)}{S\left(\mathscr{H}_{0}\left(\boldsymbol{q}^{\prime}, \boldsymbol{p}^{\prime}\right)\right)} d \boldsymbol{q}^{\prime} d \boldsymbol{p}^{\prime}=\iint R\left(\mathscr{H}_{0}(\boldsymbol{q}, \boldsymbol{p})\right) \delta f(\boldsymbol{q}, \boldsymbol{p}) d \boldsymbol{q} d \boldsymbol{p},
\end{aligned}
$$

where $S$ denotes a volume of iso- $\mathscr{H}_{0}$ set,

$$
S(E)=\iint \delta\left(E-\mathscr{H}_{0}\left(\boldsymbol{q}^{\prime}, \boldsymbol{p}^{\prime}\right)\right) d \boldsymbol{q}^{\prime} d \boldsymbol{p}^{\prime}
$$

More generally it is possible to show as in the 1D case [14] that

$$
\iint\langle a\rangle_{\mathscr{H}_{0}(\boldsymbol{q}, \boldsymbol{p})} b(\boldsymbol{q}, \boldsymbol{p}) d \boldsymbol{q} d \boldsymbol{p}=\iint a(\boldsymbol{q}, \boldsymbol{p})\langle b\rangle_{\mathscr{H}_{0}(\boldsymbol{q}, \boldsymbol{p})} d \boldsymbol{q} d \boldsymbol{p},
$$

for the functions $a$ and $b$ when $\langle a\rangle_{\mathscr{H}_{0}} b$ and $a\langle b\rangle_{\mathscr{H}_{0}}$ are integrable. Thus it has been shown that the condition (10) is equivalent to

$$
\langle\delta f\rangle_{\mathscr{H}_{0}}=0, \quad \text { for almost every }(\boldsymbol{q}, \boldsymbol{p}) .
$$

\section{LINEAR RESPONSE FORMULA}

\section{A. Implicit form of linear response}

After that the external field is exerted and the limit $t \rightarrow \infty$ is taken, the effective Hamiltonian becomes

$$
\mathscr{H}_{h}(\boldsymbol{q}, \boldsymbol{p})=\mathscr{H}_{0}(\boldsymbol{q}, \boldsymbol{p})+\delta \mathscr{V}(\boldsymbol{q})+h \Phi(\boldsymbol{q})+O\left(h^{2}\right),
$$

where the linear response $\delta \mathcal{V} \equiv \mathcal{V}[\delta f]$. Let the initial and final states be respectively as:

$$
\begin{aligned}
& f_{0}(\boldsymbol{q}, \boldsymbol{p})=F_{0}\left(\mathscr{H}_{0}(\boldsymbol{q}, \boldsymbol{p})\right)=\frac{G_{0}\left(\mathscr{H}_{0}(\boldsymbol{q}, \boldsymbol{p})\right)}{\left\langle G_{0}\right\rangle_{\mu}}, \\
& f_{h}(\boldsymbol{q}, \boldsymbol{p})=F_{h}\left(\mathscr{H}_{h}(\boldsymbol{q}, \boldsymbol{p})\right)=\frac{G_{h}\left(\mathscr{H}_{h}(\boldsymbol{q}, \boldsymbol{p})\right)}{\left\langle G_{h}\right\rangle_{\mu}},
\end{aligned}
$$

where $\langle a\rangle_{\mu}=\iint \operatorname{ad} \boldsymbol{q} d \boldsymbol{p}$ and

$$
\begin{aligned}
& G_{h}\left(\mathscr{H}_{h}\right)=G_{0}\left(\mathscr{H}_{h}\right)+h G_{1}\left(\mathscr{H}_{h}\right)+O\left(h^{2}\right) \\
& =G_{0}\left(\mathscr{H}_{0}\right)+G_{0}^{\prime}\left(\mathscr{H}_{0}\right)\left(\mathscr{H}_{h}-\mathscr{H}_{0}\right)+h G_{1}\left(\mathscr{H}_{0}\right)+O\left(h^{2}\right) .
\end{aligned}
$$

Expanding $f_{h}$ around $f_{0}$, we have

$$
\begin{aligned}
f_{h}= & f_{0}+\frac{G_{0}^{\prime}\left(\mathscr{H}_{0}\right)(\delta V+h \Phi)+G_{1}\left(\mathscr{H}_{0}\right)}{\left\langle G_{0}\left(\mathscr{H}_{0}\right)\right\rangle_{\mu}} \\
& -\frac{\left\langle G_{0}^{\prime}\left(\mathscr{H}_{0}\right)(\delta \mathcal{V}+h \Phi)+G_{1}\left(\mathscr{H}_{0}\right)\right\rangle_{\mu} G_{0}\left(\mathscr{H}_{0}\right)}{\left\langle G_{0}\left(\mathscr{H}_{0}\right)\right\rangle_{\mu}^{2}}+O\left(h^{2}\right) .
\end{aligned}
$$


We then obtain the linear response,

$$
\begin{aligned}
& \delta f \equiv F_{0}^{\prime}\left(\mathscr{H}_{0}\right)(\delta \mathcal{V}+h \Phi)+\frac{G_{1}\left(\mathscr{H}_{0}\right)}{\left\langle G_{0}\left(\mathscr{H}_{0}\right)\right\rangle_{\mu}} \\
& -\left\langle F_{0}^{\prime}\left(\mathscr{H}_{0}\right)(\delta \mathcal{V}+h \Phi)\right\rangle_{\mu} F_{0}\left(\mathscr{H}_{0}\right)-\frac{\left\langle G_{1}\left(\mathscr{H}_{0}\right)\right\rangle_{\mu} F_{0}\left(\mathscr{H}_{0}\right)}{\left\langle G_{0}\left(\mathscr{H}_{0}\right)\right\rangle_{\mu}}
\end{aligned}
$$

by taking the linear order. The function $G_{1}$ is determined so that $\langle\delta f\rangle_{\mathscr{C}_{0}}=0$, that is,

$$
\begin{aligned}
\frac{G_{1}}{\left\langle G_{0}\right\rangle_{\mu}}= & \frac{\left\langle G_{1}\right\rangle_{\mu}}{\left\langle G_{0}\right\rangle_{\mu}} F_{0}-F_{0}^{\prime}\left(\mathscr{H}_{0}\right)\langle\delta \mathcal{V}+h \Phi\rangle_{\mathscr{C}_{0}}, \\
& +F_{0}\left(\mathscr{H}_{0}\right)\left\langle F_{0}^{\prime}\left(\mathscr{H}_{0}\right)(\delta \mathcal{V}+h \Phi)\right\rangle_{\mu}
\end{aligned}
$$

where $F_{0}^{\prime}\left(\mathscr{H}_{0}\right)=G_{0}^{\prime}\left(\mathscr{H}_{0}\right) /\left\langle G_{0}\left(\mathscr{H}_{0}\right)\right\rangle_{\mu}$. The response is therefore implicitly given by

$$
\begin{aligned}
& \delta f=F_{0}^{\prime}\left(\mathscr{H}_{0}\right)\left(\delta \mathcal{V}(\boldsymbol{q})-\langle\delta \mathcal{V}(\boldsymbol{q})\rangle_{\mathscr{H}_{0}}\right) \\
& +h F_{0}^{\prime}\left(\mathscr{H}_{0}\right)\left(\Phi(\boldsymbol{q})-\langle\Phi(\boldsymbol{q})\rangle_{\mathscr{H}_{0}}\right) .
\end{aligned}
$$

Solving the implicit linear response formula (21) by using bi-orthogonal basis, we obtain explicitly the linear response taking into account the constraint conditions.

We make a comment on the case that there exist two integrals and $f_{0}$ depends on the both of them. Let $\mathscr{L}=$ $\iint L(\boldsymbol{q}, \boldsymbol{p}) f d \boldsymbol{q} d \boldsymbol{p}$ be an additional integral (the angular momentum density for example). We consider the optimization problem (6) and we add the additional constraint $\mathscr{L}=$ Const. to Eq. (6). A solution $f_{0}$ depends on $\mathscr{H}_{0}$ and $L$ as $f_{0}=F_{0}\left(\beta \mathscr{H}_{0}+v L\right)$ where $\beta$ and $v$ are Lagrangian multipliers. Thus the accessible perturbation satisfies

$$
\langle\delta f(\boldsymbol{q}, \boldsymbol{p})\rangle_{\left(\beta \mathscr{H}_{0}(\boldsymbol{q}, \boldsymbol{p})+v L(\boldsymbol{q}, \boldsymbol{p})\right)}=0,
$$

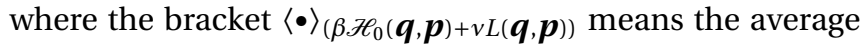
taken over iso- $\left(\beta \mathscr{H}_{0}+v L\right)$ set. It should be noted that a form of constrained perturbation depends on how $f_{0}$ depends on $\mathscr{H}_{0}$ and $L$. We should find ways to restrict the form of perturbations for each stationary state.

If $\mathscr{H}_{0}$ has three independent integrals of motion, we can use angle-action variables and can solve the linearized Vlasov equation.

\section{B. Explicit form of linear response}

We introduce the bi-orthogonal basis $[3,26,27],\left\{d_{i}(\boldsymbol{q})\right\}_{i \in \rrbracket}$

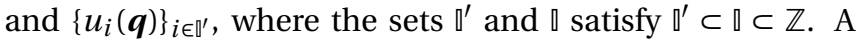
perturbation of spatial density is spanned by the base $\left\{d_{i}\right\}_{i}$,

$$
\delta \rho(\boldsymbol{q})=\int \delta f(\boldsymbol{q}, \boldsymbol{p}) d \boldsymbol{p}=\sum_{i \in \mathbb{}} a_{i}(t) d_{i}(\boldsymbol{q}) .
$$

The base $\left\{u_{i}\right\}_{i}$ is introduced as

$$
u_{i}(\boldsymbol{q}) \equiv\left(V * d_{i}\right)(\boldsymbol{q}) \equiv \int V\left(\boldsymbol{q}-\boldsymbol{q}^{\prime}\right) d_{i}\left(\boldsymbol{q}^{\prime}\right) d \boldsymbol{q}^{\prime},
$$

and the orthogonal relation

$$
\int d_{i}(\boldsymbol{q}) \bar{u}_{j}(\boldsymbol{q}) d \boldsymbol{q}=\lambda_{j} \delta_{i j}
$$

holds, where $\lambda_{i} \neq 0$ when $i \in \mathbb{Q}^{\prime}$ and it vanishes otherwise, and $\delta_{i j}$ is the Kronecker delta. The upper bar denotes complex conjugate. Integrating the terms including $\delta f$ or $\delta V$ in Eq. (21) with respect to $\boldsymbol{p}$, we have

$$
\begin{aligned}
& \int \delta f d \boldsymbol{p}-\int F_{0}^{\prime}\left(\mathscr{H}_{0}\right)\left(\delta \mathcal{V}(\boldsymbol{q})-\langle\delta \mathcal{V}(\boldsymbol{q})\rangle_{\mathscr{H}_{0}}\right) d \boldsymbol{p} \\
& =\sum_{i \in \llbracket} a_{i}\left[d_{i}-\int F_{0}^{\prime}\left(\mathscr{H}_{0}\right)\left(u_{i}(\boldsymbol{q})-\left\langle u_{i}(\boldsymbol{q})\right\rangle_{\mathscr{H}_{0}}\right) d \boldsymbol{p}\right] .
\end{aligned}
$$

Multiplying $\bar{u}_{j}$ to both sides and integrating them with respect to $\boldsymbol{q}$, we have,

$$
\begin{aligned}
& \sum_{i \in \mathbb{1}^{\prime}} a_{i}\left[\lambda_{j} \delta_{i j}-\int F_{0}^{\prime}\left(\mathscr{H}_{0}\right)\left(u_{i} \bar{u}_{j}-\left\langle u_{i}\right\rangle_{\mathscr{H}_{0}}\left\langle\bar{u}_{j}\right\rangle_{\mathscr{H}_{0}}\right) d \boldsymbol{p} d \boldsymbol{q}\right] \\
& =\sum_{i \in \mathbb{D}^{\prime}}\left[\lambda_{j} \delta_{j i}-\int F_{0}^{\prime}\left(\mathscr{H}_{0}\right)\left(\bar{u}_{j} u_{i}-\left\langle\bar{u}_{j}\right\rangle_{\mathscr{H}_{0}}\left\langle u_{i}\right\rangle_{\mathscr{H}_{0}}\right) d \boldsymbol{p} d \boldsymbol{q}\right] a_{i}
\end{aligned}
$$

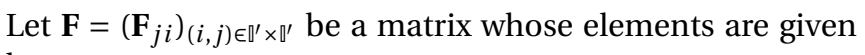
by

$$
\mathbf{F}_{j i}=\int F_{0}^{\prime}\left(\mathscr{H}_{0}\right)\left(\bar{u}_{j} u_{i}-\left\langle\bar{u}_{j}\right\rangle_{\mathscr{H}_{0}}\left\langle u_{i}\right\rangle_{\mathscr{H}_{0}}\right) d \boldsymbol{p} d \boldsymbol{q} .
$$

We further assume that the term coupling with external force can be expanded as

$$
\Phi(\boldsymbol{q})=\sum_{i \in \mathbb{\natural}^{\prime}} b_{i} u_{i}(\boldsymbol{q})
$$

We then have

$$
\begin{aligned}
& h \int F_{0}^{\prime}\left(\mathscr{H}_{0}\right)\left(\Phi(\boldsymbol{q})-\langle\Phi(\boldsymbol{q})\rangle_{\mathscr{H}_{0}}\right) d \boldsymbol{p} \\
& =h \sum_{i \in \mathbb{J}^{\prime}} b_{i} \int F_{0}^{\prime}\left(\mathscr{H}_{0}\right)\left(u_{i}(\boldsymbol{q})-\left\langle u_{i}(\boldsymbol{q})\right\rangle_{\mathscr{H}_{0}}\right) d \boldsymbol{p},
\end{aligned}
$$

by integrating the term coming from external force in Eq. (21) with respect to $\boldsymbol{p}$. Multiplying $\bar{u}_{j}$ to it and integrating with respect to $\boldsymbol{q}$, we have, (as we have already done)

$$
h \sum_{i \in \mathbb{\rrbracket}^{\prime}} \mathbf{F}_{j i} b_{i} .
$$

Combining Eqs. (27) and (31), we get the linear equation determining $\left\{a_{i}\right\}_{i \in \mathbb{D}^{\prime}}$,

$$
\sum_{j \in \rrbracket^{\prime}}\left(\lambda_{j} \delta_{i j}-\mathbf{F}_{i j}\right) a_{j}=h \sum_{j \in \rrbracket^{\prime}} \mathbf{F}_{i j} b_{j}
$$

Introducing symbols $\mathbf{x}=\left(x_{i}\right)_{i \in \mathbb{\rrbracket}^{\prime}}$ for $x=a, b$ and $\Lambda=$ $\operatorname{diag}\left(\lambda_{i}\right)_{i \in \mathbb{D}^{\prime}}$, we can simplify the equation as follows:

$$
\left(\mathbf{1}-\Lambda^{-1} \mathbf{F}\right) \mathbf{a}=h \Lambda^{-1} \mathbf{F b},
$$


and it is solved as

$$
\mathbf{a}=h(\mathbf{1}-\mathbf{F})^{-1} \mathbf{F b}=h \mathbf{D}^{-1}(1-\mathbf{D}) \mathbf{b},
$$

where $\mathbf{1}$ denotes the unit matrix and $\mathbf{D}=\mathbf{1}-\Lambda^{-1} \mathbf{F}$. The maximal-eigenvalue of $\mathbf{D}$ is zero when $f_{0}$ might be marginally stable, and corresponds to the critical point. When we apply Eq. (34) to 1D systems, This explicit response formula formally coincides with what is derived in Ref. [11].

\section{EXAMPLES: HAMILTONIAN MEAN-FIELD MODELS}

\section{A. One dimensional case}

Let us examine the proposed theory by use of the Hamiltonian mean-field (HMF) model whose Hamiltonian is

$$
H=\sum_{i=1}^{N} \frac{p_{i}^{2}}{2}-\frac{1}{2 N} \sum_{i \neq j} \cos \left(q_{i}-q_{j}\right)-h \sum_{i=1}^{N} \cos q_{i} .
$$

where $h$ is an external field, $p_{i} \in \mathbb{R}, q_{i} \in[-\pi, \pi)$ for $i=$ $1,2, \cdots, N$. In the equilibrium state, this model shows second order phase transition at the temperature $T=0.5$, where the Boltzmann's constant $k_{\mathrm{B}}=1$. By use of the linear and nonlinear response formulae, the critical phenomena in the equilibrium state and QSSs for the isolated HMF model are investigated and it is shown that the Casimir constraints bring about the non-classical critical exponents [13-15]. We here check that the present method yields the linear response formula same with the previously obtained one for the 1D HMF model. The effective Hamiltonian is

$$
\mathscr{H}=\frac{p^{2}}{2}-\iint \cos \left(q-q^{\prime}\right) f\left(q^{\prime}, p^{\prime}, t\right) d q^{\prime} d p^{\prime}-h \cos q .
$$

Applying Eq. (21) for the HMF model, we can derive the linear response formula

$$
\delta f=(-\delta M-h) F_{0}^{\prime}\left(\mathscr{H}_{0}\right)\left(\cos q-\langle\cos q\rangle_{\mathscr{H}_{0}}\right),
$$

where $\delta M=\iint \cos q \delta f d q d p$. Multiplying $\cos q$ to both sides and integrating over the $\mu$-space, we obtain the linear response as

$$
\delta M=\frac{1-D}{D} h
$$

where

$$
D=1+\iint F^{\prime}\left(\mathscr{H}_{0}\right)\left(\cos ^{2} q-\langle\cos q\rangle_{\mathscr{H}_{0}}^{2}\right) d q d p .
$$

This is equivalent to the linear response formula obtained in Ref. [13].

For more general 1D systems it is obvious that Eq. (21) is equivalent to the linear order of the nonlinear response formula derived in Ref. [15].

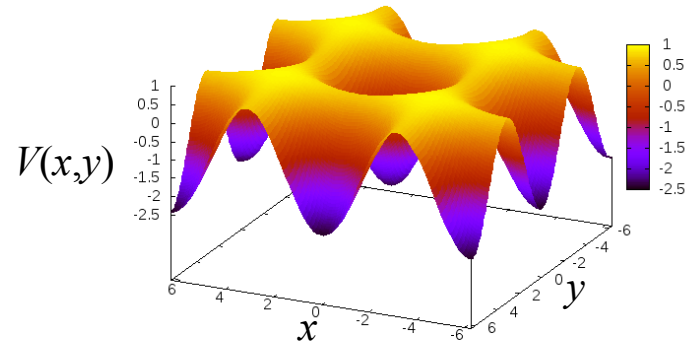

FIG. 1. (Color online) The effective potential of the Hamiltonian $(41)$. A minimum point is at $(0,0)$ and $\min V=-2 M-P$. Saddle points are at $( \pm \pi, 0)$ and $(0, \pm \pi)$ and $V=P$. A maximum point are on $( \pm \pi, \pm \pi)$ and $\max \mathscr{V}=2 M-P$, where $M>P>0$.

\section{B. Two dimensional case}

We next examine our theory in the $2 \mathrm{D}$ system whose Hamiltonian is

$$
\begin{array}{r}
H=\sum_{i=1}^{N} \frac{\left\|\boldsymbol{p}_{i}\right\|^{2}}{2}-h_{x} \sum_{i=1}^{N} \cos x_{i}-h_{y} \sum_{i=1}^{N} \cos y_{i} \\
-\frac{1}{2 N} \sum_{i \neq j}\left[\cos \left(x_{i}-x_{j}\right)+\cos \left(y_{i}-y_{j}\right)\right. \\
\left.+\cos \left(x_{i}-x_{j}\right) \cos \left(y_{i}-y_{j}\right)\right],
\end{array}
$$

where $\boldsymbol{q}_{i}=\left(x_{i}, y_{i}\right) \in[-\pi, \pi)^{2}$ for $i=1,2, \cdots, N[28,29]$. Let us assume the initial QSS $f_{0}$ with $h_{x}=h_{y}=0$ is even with respect to both $x$ and $y$. Then, the effective Hamiltonian is

$$
\begin{aligned}
\mathscr{H}_{0} & =\frac{p_{x}^{2}+p_{y}^{2}}{2}+\mathcal{V}(x, y), \\
\mathcal{V}(x, y) & =-M_{x} \cos x-M_{y} \cos y-P_{\operatorname{cc}} \cos x \cos y,
\end{aligned}
$$

where

$$
\begin{aligned}
& M_{x}=\iint \cos x \rho(\boldsymbol{q}) d \boldsymbol{q}, \quad M_{y}=\iint \cos y \rho(\boldsymbol{q}) d \boldsymbol{q}, \\
& P_{\mathrm{cc}}=\iint \cos x \cos y \rho(\boldsymbol{q}) d \boldsymbol{q},
\end{aligned}
$$

and where $\rho(\boldsymbol{q})=\iint f(\boldsymbol{q}, \boldsymbol{p}) d \boldsymbol{p}$. The effective potential is shown in Fig. 1.

To compute the linear response of the macroscopic observables $M_{x}, M_{y}$, and $P_{\mathrm{cc}}$ to the external field $h_{x}=h_{y}=$ $h$, it is necessary to compute $\langle\cos x\rangle_{\mathscr{H}_{0}},\langle\cos y\rangle_{\mathscr{H}_{0}}$, and $\langle\cos x \cos y\rangle_{\mathscr{H}_{0}}$. We here set $M_{x}=M_{y}=M$ and $P_{\mathrm{cc}}=P$.

For any smooth function $g$ depending only on $\boldsymbol{q},\langle g\rangle_{E}$ is expressed as follows (see derivation for Appendix B.)

$$
\begin{aligned}
\langle g\rangle_{E} & =\int_{\mathbf{R}^{2}} d \boldsymbol{p} \int_{[-\pi, \pi)^{2}} g(\boldsymbol{q}) \delta(\mathscr{H}(\boldsymbol{q}, \boldsymbol{p})-E) d \boldsymbol{q} \\
& =2 \pi \int_{[-\pi, \pi)^{2}} g(\boldsymbol{q}) \Theta(E-\mathcal{V}(x, y)) d \boldsymbol{q} .
\end{aligned}
$$


Since $\mathscr{H}_{0}$ is even with respect to both $x$ and $y$, we have

$$
\begin{gathered}
\langle\sin x\rangle_{\mathscr{H}_{0}}=\langle\sin y\rangle_{\mathscr{H}_{0}}=\langle\sin x \cos y\rangle_{\mathscr{H}_{0}} \\
=\langle\sin x \sin y\rangle_{\mathscr{H}_{0}}=\langle\cos x \sin y\rangle_{\mathscr{H}_{0}}=0 .
\end{gathered}
$$

When $\mathscr{H}_{0}>2 M-P=\max \mathcal{V}(x, y)$, we have

$$
\langle\cos x\rangle_{\mathscr{H}_{0}}=\langle\cos y\rangle_{\mathscr{H}_{0}}=\langle\cos x \cos y\rangle_{\mathscr{H}_{0}}=0 .
$$

Then we have to compute $\langle\cos x\rangle_{\mathscr{H}_{0}},\langle\cos y\rangle_{\mathscr{H}_{0}}$, and $\langle\cos x \cos y\rangle_{\mathscr{H}_{0}}$ when $\mathscr{H}_{0}<2 M-P$, and these are exhibited in Appendix C. We next derive an explicit form of linear responses,

$$
\begin{array}{r}
\delta M_{x}=\iint \cos x \delta f d \boldsymbol{q} d \boldsymbol{p}, \quad \delta M_{y}=\iint \cos y \delta f d \boldsymbol{q} d \boldsymbol{p}, \\
\delta P_{\mathrm{cc}}=\iint \cos x \cos y \delta f d \boldsymbol{q} d \boldsymbol{p} .
\end{array}
$$

The following notations are introduced for simplicity;

$$
\begin{aligned}
G_{1} & =-\iint F_{0}^{\prime}\left(\mathscr{H}_{0}\right)\left(\cos ^{2} x-\langle\cos x\rangle_{\mathscr{H}_{0}}^{2}\right) d \boldsymbol{q} d \boldsymbol{p}=-\iint F_{0}^{\prime}\left(\mathscr{H}_{0}\right)\left(\cos ^{2} y-\langle\cos y\rangle_{\mathscr{H}_{0}}^{2}\right) d \boldsymbol{q} d \boldsymbol{p}, \\
G_{2} & =-\iint F_{0}^{\prime}\left(\mathscr{H}_{0}\right)\left(\cos x \cos y-\langle\cos x\rangle_{\mathscr{H}_{0}}\langle\cos y\rangle_{\mathcal{H}_{0}}\right) d \boldsymbol{q} d \boldsymbol{p}=-\iint F_{0}^{\prime}\left(\mathscr{H}_{0}\right)\left(\cos x \cos y-\langle\cos x\rangle_{\mathscr{H}_{0}}^{2}\right) d \boldsymbol{q} d \boldsymbol{p}, \\
G_{3} & =-\iint F_{0}^{\prime}\left(\mathscr{H}_{0}\right)\left(\cos ^{2} x \cos y-\langle\cos x \cos y\rangle_{\mathscr{H}_{0}}\langle\cos x\rangle_{\mathcal{H}_{0}}\right) d \boldsymbol{q} d \boldsymbol{p} \\
& =-\iint F_{0}^{\prime}\left(\mathscr{H}_{0}\right)\left(\cos x \cos ^{2} y-\langle\cos x \cos y\rangle_{\mathscr{H}_{0}}\langle\cos y\rangle_{\mathcal{H}_{0}}\right) d \boldsymbol{q} d \boldsymbol{p}, \\
G_{4} & =-\iint F_{0}^{\prime}\left(\mathscr{H}_{0}\right)\left(\cos ^{2} x \cos ^{2} y-\langle\cos x \cos y\rangle_{\mathscr{H}_{0}}^{2}\right) d \boldsymbol{q} d \boldsymbol{p} .
\end{aligned}
$$

By use of them and Eq. (21), we have

$$
\left(\begin{array}{ccc}
1-G_{1} & -G_{2} & -G_{3} \\
-G_{2} & 1-G_{1} & -G_{3} \\
-G_{3} & -G_{3} & 1-G_{4}
\end{array}\right)\left(\begin{array}{l}
\delta M_{x} \\
\delta M_{y} \\
\delta P_{\mathrm{cc}}
\end{array}\right)=\left(\begin{array}{c}
h_{x} G_{1}+h_{y} G_{2} \\
h_{x} G_{2}+h_{y} G_{1} \\
\left(h_{x}+h_{y}\right) G_{3}
\end{array}\right)
$$

We therefore obtain the explicit linear response formula as follows:

$$
\begin{aligned}
& \delta M_{x}=\chi_{1} h_{x}+\chi_{2} h_{y}, \quad \delta M_{y}=\chi_{2} h_{x}+\chi_{1} h_{y}, \\
& \delta P_{\mathrm{cc}}=\chi_{3}\left(h_{x}+h_{y}\right),
\end{aligned}
$$

where explicit expressions of $\chi_{1}, \chi_{2}$, and $\chi_{3}$ are

$$
\begin{aligned}
& \chi_{1}=\frac{1}{\operatorname{det} \mathbf{G}}\left(G_{1}-G_{1}^{2}-G_{1} G_{4}+G_{2}^{2}+G_{3}^{2}\right. \\
& \left.+G_{1}^{2} G_{4}-2 G_{1} G_{3}^{2}+2 G_{2} G_{3}^{2}-G_{2}^{2} G_{4}\right), \\
& \chi_{2}=\frac{1}{\operatorname{det} \mathbf{G}}\left(G_{2}+G_{3}^{2}-G_{2} G_{4}\right), \\
& \chi_{3}=\frac{1}{\operatorname{det} \mathbf{G}} G_{3}\left(1-G_{1}+G_{2}\right) \\
& =\frac{G_{3}}{1-G_{1}-G_{2}-G_{4}+G_{1} G_{4}+G_{2} G_{4}-2 G_{3}^{2}},
\end{aligned}
$$

respectively, where the determinant of $\mathbf{G}$, the matrix in the left hand side of Eq. (48), is

$$
\begin{aligned}
\operatorname{det} \mathbf{G}= & \left(1-G_{1}+G_{2}\right) \\
& \times\left(1-G_{1}-G_{2}-G_{4}+G_{1} G_{4}+G_{2} G_{4}-2 G_{3}^{2}\right) .
\end{aligned}
$$

A way to compute terms including $\langle\cos x\rangle_{\mathscr{H}_{0}},\langle\cos y\rangle_{\mathscr{H}_{0}}$, and $\langle\cos x \cos y\rangle_{\mathscr{H}_{0}}$ is exhibited in Appendix D.

When $F_{0}\left(\mathscr{H}_{0}\right)$ is spatially homogeneous, that is, $M=P=$ 0 , we have $G_{2}=G_{3}=0$ and $G_{1}$ and $G_{4}\left(1-G_{1}<1-G_{4}\right.$ when $M=P=0$ ) do not vanish. Thus the susceptibilities are

$$
\chi_{1}=\frac{G_{1}}{1-G_{1}}, \quad \chi_{2}=\chi_{3}=0
$$

in the disordered phase.

We numerically confirm the linear response formula. The initial state is the Maxwell-Boltzmann type:

$$
f_{\mathrm{MB}}(\boldsymbol{q}, \boldsymbol{p})=\frac{\exp \left(-\mathscr{H}_{0} / T\right)}{\left\langle\exp \left(-\mathscr{H}_{0} / T\right)\right\rangle_{\mu}} .
$$

This system shows the first order phase transition [28] and there is no (meta-)stable homogeneous state with $T<0.5$. The initial values of order parameters for $T=0.3$ and 0.4 are exhibited in Table I. The external field $h_{x}=h_{y}=h$ is exerted. Theoretically obtained susceptibilities are exhibited in Table I when the temperature $T=0.3$ and 0.4 , so that the initial equilibria are spatially inhomogeneous. We integrate an equation of motion derived from the Hamiltonian (40) by using a fourth order symplectic integrator [30], and compute the order parameters of $N$ body systems, given respec-

TABLE I. Initial equilibria and zero-field susceptibilities

\begin{tabular}{c|rr|rr}
\hline \hline$T$ & $M$ & $P$ & $d \delta M_{x / y} /\left.d h\right|_{h=0} d \delta P_{\mathrm{cc}} /\left.d h\right|_{h=0}$ \\
\hline 0.3 & 0.90223 & 0.81556 & 0.034428 & 0.059089 \\
0.4 & 0.84269 & 0.71910 & 0.071298 & 0.099709 \\
\hline \hline
\end{tabular}


tively by

$$
\begin{gathered}
M_{x \mathrm{c}}^{N}(t, h)=\frac{1}{N} \sum_{i=1}^{N} \cos x_{i}(t, h), \\
M_{y \mathrm{c}}^{N}(t, h)=\frac{1}{N} \sum_{i=1}^{N} \cos y_{i}(t, h), \\
M_{x \mathrm{~s}}^{N}(t, h)=\frac{1}{N} \sum_{i=1}^{N} \sin x_{i}(t, h), \\
M_{y \mathrm{~s}}^{N}(t, h)=\frac{1}{N} \sum_{i=1}^{N} \sin y_{i}(t, h), \\
P_{\mathrm{cc}}^{N}(t, h)=\frac{1}{N} \sum_{i=1}^{N} \cos x_{i}(t, h) \cos y_{i}(t, h), \\
P_{\mathrm{cs}}^{N}(t, h)=\frac{1}{N} \sum_{i=1}^{N} \cos x_{i}(t, h) \sin y_{i}(t, h), \\
P_{\mathrm{sc}}^{N}(t, h)=\frac{1}{N} \sum_{i=1}^{N} \sin x_{i}(t, h) \cos y_{i}(t, h), \\
P_{\mathrm{ss}}^{N}(t, h)=\frac{1}{N} \sum_{i=1}^{N} \sin x_{i}(t, h) \sin y_{i}(t, h),
\end{gathered}
$$

for null amplitude $h=0$ and non-zero $h$. We compare the theoretically obtained linear response $\delta M_{x}, \delta M_{y}$, and $\delta P_{\mathrm{cc}}$ with the numerically obtained responses given respectively by

$$
\begin{aligned}
& \delta M_{x}^{N}(h)=\bar{M}_{x}^{N}(h)-\bar{M}_{x}^{N}(0), \\
& \delta M_{y}^{N}(h)=\bar{M}_{y}^{N}(h)-\bar{M}_{y}^{N}(0), \\
& \delta P_{x y}^{N}(h)=\bar{P}_{x y}^{N}(h)-\bar{P}_{x y}^{N}(0),
\end{aligned}
$$

where $M_{x}^{N}, M_{y}^{N}$, and $P_{x y}^{N}$ are given by

$$
\begin{aligned}
& M_{x}^{N}=\sqrt{M_{x \mathrm{c}}^{N^{2}}+M_{x \mathrm{~s}}^{N^{2}}}, \\
& M_{y}^{N}=\sqrt{{M_{y \mathrm{c}}^{N}+M_{y \mathrm{~s}}^{N^{2}}}^{2}} \\
& P_{x y}^{N}=\sqrt{P_{\mathrm{cc}}^{N^{2}}+P_{\mathrm{cs}}^{N^{2}}+P_{\mathrm{sc}}^{N^{2}}+P_{\mathrm{ss}}^{N^{2}}},
\end{aligned}
$$

and where upper bars in Eq. (58) denote the time average

$$
\bar{M}_{x \mathrm{c}}^{N}(h)=\frac{1}{\tau} \int_{t_{0}}^{t_{0}+\tau} M_{x \mathrm{c}}^{N}(t, h) d t .
$$

For the 2D HMF model there is error between $\bar{M}_{x}^{N}(0)$ and $M$ which is a solution to the self-consistent equation. We then focus on the difference $\bar{M}_{x}^{N}(h)-\bar{M}_{x}^{N}(0)$ rather than $\bar{M}_{x}^{N}(h)-M$. We set $t_{0}=200, \tau=200, N=4 \times 10^{6}$, and the time step $\delta t=0.05$. Figure 2 shows that these $t_{0}$ and $\tau$ are appropriate, and Fig. 3 shows that the numerically obtained results confirm the theory. (a)

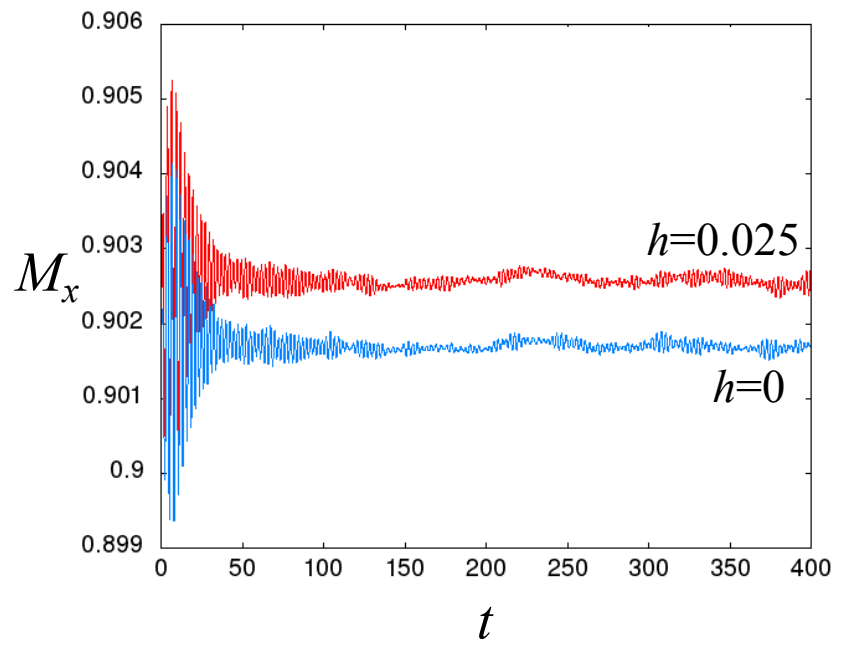

(b)

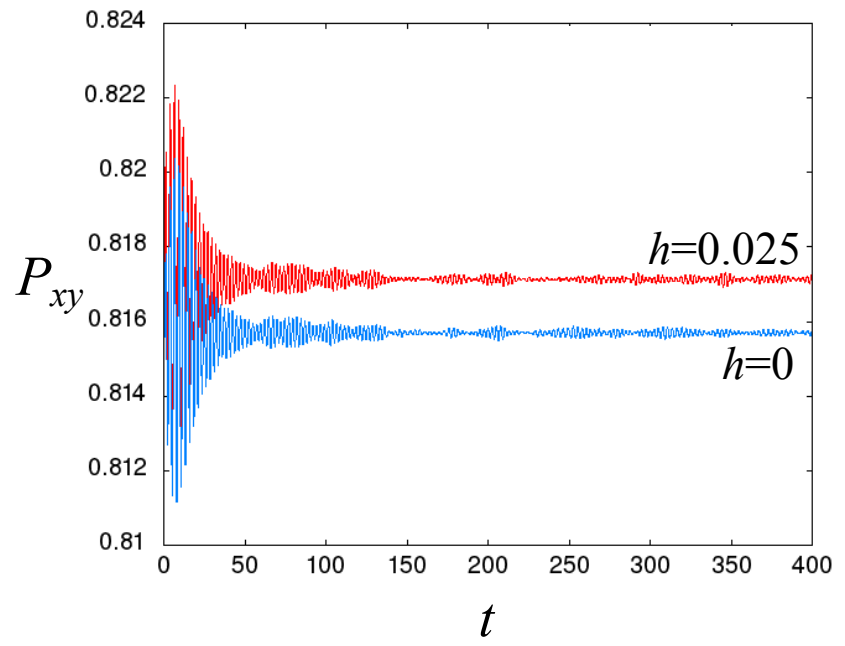

FIG. 2. (Color online) Time series of order parameters: The panel (a) is the time series for $M_{x}$ and the panel (b) for $P_{x y}$. The temperature $T=0.3$, the number of particles $N=4 \times 10^{6}$, and the time step $\delta t=0.05$. For each panel, the upper (red) curve is for $h=0.025$ and the lower (blue) one for $h=0$.

\section{NONLINEAR RESPONSE FORMULA}

The nonlinear response formula [14] which is called the rearrangement formula in Ref. [22] keeps the Casimir invariants within an order of the T-linearization, the linearization around an asymptotic (A-) state $f_{\mathrm{A}}(\boldsymbol{q}, \boldsymbol{p})=\lim _{t \rightarrow \infty} f(\boldsymbol{q}, \boldsymbol{p}, t)$ assumed to be stationary. We derive the nonlinear response formula via the same strategy for deriving the linear response formula in the present article. We assume that the asymptotic effective Hamiltonian

$$
\mathscr{H}_{\mathrm{A}}=\|\boldsymbol{p}\|^{2} / 2+\mathcal{V}_{\mathrm{A}}+h \Phi, \quad \mathcal{V}_{\mathrm{A}}=\mathcal{V}\left[f_{\mathrm{A}}\right],
$$

have only one integral of a single particle motion and $f_{\mathrm{A}}$ depends only on $\mathscr{H}_{\mathrm{A}}$. Further $f_{\mathrm{A}}=F_{\mathrm{A}}\left(\mathscr{H}_{\mathrm{A}}\right)$ is assumed to be monotonically decreasing with respect to $\mathscr{H}_{\mathrm{A}}$. Under 
(a)

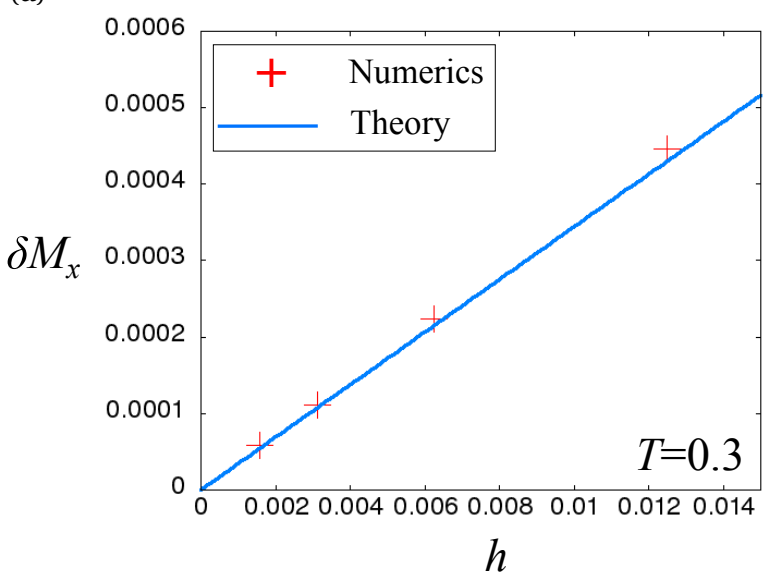

(c)

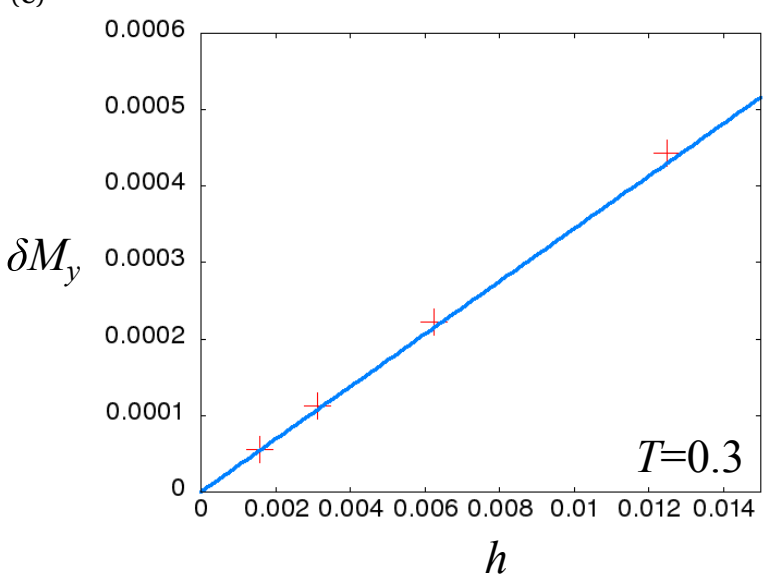

(e)

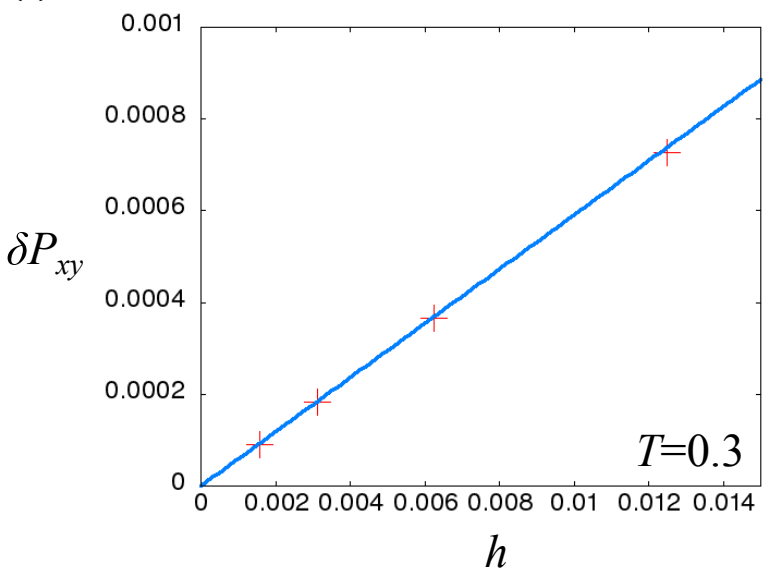

(b)

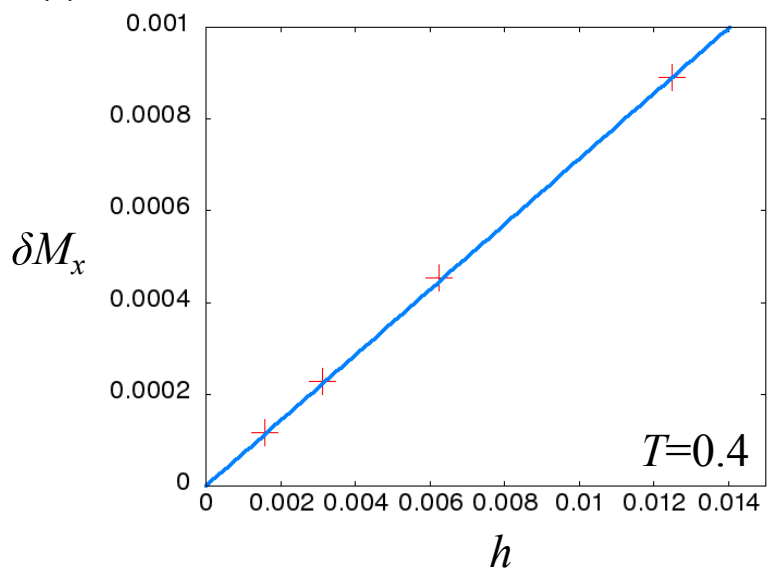

(d)

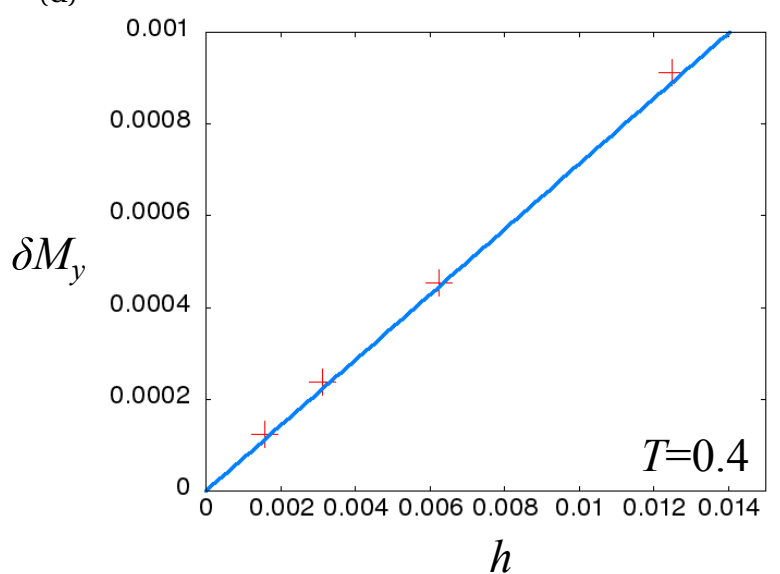

(f)

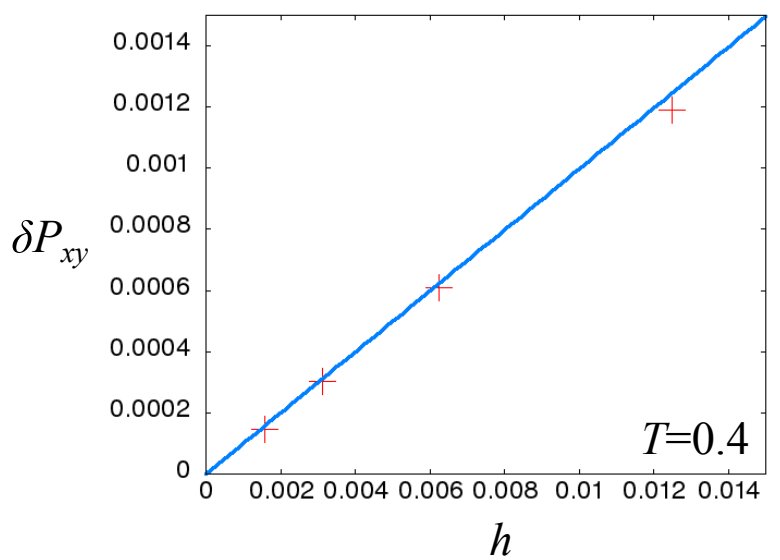

FIG. 3. (Color online) $\delta M_{x}, \delta M_{y}$, and $\delta P_{x y}$ as functions of $h$. The lines are the linear responses obtained theoretically and the crosses are responses obtained numerically. We set temperature of the initial states as $T=0.3$ (Left column, panels (a, c, e)) and $T=0.4$ (Right column, panels (b, d, f)), and a number of particles $N=4 \times 10^{6}$ and the time step $\delta t=0.05$.

these assumptions, expanding Eq. (8) around $f_{\mathrm{A}}$ as done in Sec. III, the constraint condition coming from Casimir invariants within an order of T-linearization can be expressed as

$$
\iint R\left(\mathscr{H}_{\mathrm{A}}(\boldsymbol{q}, \boldsymbol{p})\right)\left(f_{0}(\boldsymbol{q}, \boldsymbol{p})-f_{\mathrm{A}}(\boldsymbol{q}, \boldsymbol{p})\right) d \boldsymbol{q} d \boldsymbol{p}=0,
$$

for any smooth function $R$ on $\mathbb{R}$. By use of Eq. (13), it is shown that Eq. (62) holds true if and only if

$$
f_{\mathrm{A}}=\left\langle f_{0}\right\rangle_{\mathscr{H}_{\mathrm{A}}}
$$


for almost every $(\boldsymbol{q}, \boldsymbol{p})$ in the $\mu$-space. Deriving a selfconsistent equation

$$
\mathcal{V}_{\mathrm{A}}(\boldsymbol{q})=\iint V\left(\boldsymbol{q}-\boldsymbol{q}^{\prime}\right)\left\langle f_{0}\right\rangle_{\mathscr{H}_{\mathrm{A}}\left(\boldsymbol{q}^{\prime}, \boldsymbol{p}^{\prime}\right)} d \boldsymbol{q}^{\prime} d \boldsymbol{p}^{\prime}
$$

from Eq. (63) and solving it, one can obtain $\mathscr{H}_{\mathrm{A}}$ and the nonlinear response $\delta f=f_{\mathrm{A}}-f_{0}$.

Is it possible to derive Eq. (63) for the multi-dimensional systems as done in Refs. [14, 15]? There is some difficulty to derive the same formula from the T-linearization method for the multi-dimensional systems. To see this, let us exhibit a sketch of this T-linearization method (see Refs. [14, 20-22] for details.) We firstly divide $f(\boldsymbol{q}, \boldsymbol{p}, t)$ in two ways: One is the naive perturbation decomposition,

$$
f=f_{0}+g_{\mathrm{p}}
$$

where $g_{\mathrm{p}}$ is the perturbation around $f_{0}$, and the other one is the asymptotic-transient (AT) decomposition

$$
f=f_{\mathrm{A}}+g_{\mathrm{T}},
$$

where $g_{\mathrm{T}}$ is the T-term satisfying $\lim _{t \rightarrow \infty} g_{\mathrm{T}}=0$. According to the AT decomposition, the potential is also decomposed as

$$
\begin{aligned}
\mathcal{V}[f]+h(t) \Psi & =\mathcal{V}_{\mathrm{A}}+\mathcal{V}_{\mathrm{T}}+h \Phi, \\
\mathcal{V}_{\mathrm{T}} & =\mathcal{V}\left[g_{\mathrm{T}}\right]+(h(t)-h) \Phi .
\end{aligned}
$$

Substituting Eqs. (65) and (67) into the Vlasov equation, and omitting the nonlinear term coupling with the T-field $\mathcal{V}_{\mathrm{T}}$, we have the T-linearized Vlasov equation

$$
\frac{\partial f}{\partial t}+\left\{\mathscr{H}_{\mathrm{A}}, f\right\}+\left\{\mathcal{V}_{\mathrm{T}}, f_{0}\right\}=0
$$

It should be noted that the nonlinearity still remains in the term $\left\{\mathscr{H}_{\mathrm{A}}, f\right\}$. A solution $f_{\mathrm{TL}}$ to the T-linearized equation is implicitly given by

$$
\begin{aligned}
f_{\mathrm{TL}}(\boldsymbol{q}, \boldsymbol{p}, t) & =f_{\mathrm{ON}}(\boldsymbol{q}, \boldsymbol{p}, t)+f_{\mathrm{LA}}(\boldsymbol{q}, \boldsymbol{p}, t), \\
f_{\mathrm{ON}}(\boldsymbol{q}, \boldsymbol{p}, t) & =e^{-t\left\{\mathcal{X}_{\mathrm{A}}, \bullet\right\}} f_{0}(\boldsymbol{q}, \boldsymbol{p}), \\
f_{\mathrm{LA}}(\boldsymbol{q}, \boldsymbol{p}, t) & =-\int_{0}^{t} e^{-(t-s)\left\{\mathcal{X}_{\mathrm{A}}, \bullet\right\}}\left(\boldsymbol{F}_{\mathrm{T}} \cdot \frac{\partial f_{0}}{\partial \boldsymbol{p}}\right) d s,
\end{aligned}
$$

where we introduce the operator $\left\{\mathscr{H}_{\mathrm{A}}, \bullet\right\} a=\left\{\mathscr{H}_{\mathrm{A}}, a\right\}$ for any function $a(\boldsymbol{q}, \boldsymbol{p})$, and $\boldsymbol{F}_{\mathrm{T}}=-\partial V_{\mathrm{T}} / \partial \boldsymbol{q}$. The terms $f_{\mathrm{ON}}$ and $f_{\mathrm{LA}}$ are called O'Neil term and Landau term respectively [20, 21].

When the asymptotic stationary state $f_{\mathrm{A}}$ exists, it can be picked by taking the long-time average of $f_{\mathrm{TL}}$, and we have

$$
f_{\mathrm{A}}(\boldsymbol{q}, \boldsymbol{p})=\lim _{\tau \rightarrow \infty} \frac{1}{\tau} \int_{0}^{\tau} f_{\mathrm{TL}}(\boldsymbol{q}, \boldsymbol{p}, t) d t
$$

within an order of the T-lineatization method. Then, our next job is to compute the long-time average of $f_{\mathrm{ON}}$ and $f_{\mathrm{LA}}$, but there are several difficulties to this for the multidimensional systems.
In the $1 \mathrm{D}$ systems, it is shown that

$$
\lim _{\tau \rightarrow \infty} \frac{1}{\tau} \int_{0}^{\tau} e^{-t\left\{\mathscr{H}_{\mathrm{A}}, \bullet\right\}} a(\boldsymbol{q}, \boldsymbol{p}) d t=\langle a\rangle_{\mathscr{H}_{\mathrm{A}}(\boldsymbol{q}, \boldsymbol{p}),}
$$

by use of the angle-action variables of $\mathscr{H}_{\mathrm{A}}[20,21]$. However, in our case, the angle-action variables cannot be constructed, so that it is unclear that this ergodic like formula holds true or not.

There is another problem, slowly algebraic damping of Tforce-field $\boldsymbol{F}_{\mathrm{T}}$. In Ref. [14] we use the fact that $\boldsymbol{F}_{\mathrm{T}}$ damps rapidly ( $\sim t^{-v}$ with $v \geq 2$ ) for the 1D systems [26] and

$$
\lim _{t \rightarrow \infty} \int_{t}^{\infty} \boldsymbol{F}_{\mathrm{T}}(t) d t=\mathbf{0}
$$

when we compute $\lim _{t \rightarrow 0} f_{\mathrm{LA}}$. Meanwhile, in the multidimensional Vlasov systems [27] and the 2D Euler equations [31], the T-force-field $\boldsymbol{F}_{\mathrm{T}}$ damps as or slower than $t^{-1}$, so that the integral $\int_{0}^{\infty} \boldsymbol{F}_{\mathrm{T}}(t) d t$ is not defined in the $L^{1}$ meaning apparently. Sometimes, the transient part is asymptotically $\boldsymbol{F}_{\mathrm{T}}=e^{-i \Omega t} t^{-\gamma}(0<\gamma \leq 1)$ with $\Omega \neq 0$, and the integral $\int_{0}^{\infty} \boldsymbol{F}_{\mathrm{T}}(t) d t$ exists in the Riemannian meaning. In this case, one should be more careful when one computes the integrals and takes the limit. It should be remarked that there exists a case that $\Omega=0$ [27], so that it should be checked for each system.

The relation between the nonlinear response formula obtained by considering the constraint conditions and a solution to the T-linearized Vlasov equation might be an interesting future problem

\section{SUMMARY AND PERSPECTIVE}

The linear response formula has been derived without use of the analytic solution of the single particle orbit or the angle-action variables of the effective Hamiltonian. The present method improves the generalized linear response formula obtained in Ref. [16] when the back ground density function is a monotonically decreasing function of the effective Hamiltonian $\mathscr{H}_{0}$. The response formula (21) results in the one obtained in the previous studies for 1D systems $[10,11,13,15]$, and is numerically confirmed by use of the 2D HMF model. Further the nonlinear response formula [14] has been derived via the same strategy, when the asymptotic solution $f_{\mathrm{A}}$ to the T-linearized Vlasov equation is monotonically decreasing function of the effective Hamilto$\operatorname{nian} \mathscr{H}_{\mathrm{A}}$.

The nonlinear response theory based on the Tlinearization method deals with the nonlinearity of order $O\left(h^{v}\right)$ with $1<v<2$ [14]. It should be noted that it is difficult to obtain the nonlinear response of order higher than $O\left(h^{2}\right)$ successively via the proposed method, so that the error $O\left(h^{2}\right)$ is unavoidable up to now. Let $\delta f_{n}$ be a response of order $O\left(h^{n}\right)$ for $n \in \mathbb{Z}$. The condition Eq. (10) in 
the nonlinear regime $O\left(h^{2}\right)$ is written as

$$
\begin{aligned}
0 & =\iint\left(R_{1}\left(\mathscr{H}_{0}\right) \delta f_{2}+R_{2}\left(\mathscr{H}_{0}\right) \delta f_{1}^{2}\right) d \boldsymbol{q} d \boldsymbol{p} \\
& =\iint\left(R_{1}\left(\mathscr{H}_{0}\right)\left\langle\delta f_{2}\right\rangle_{\mathscr{H}_{0}}+R_{2}\left(\mathscr{H}_{0}\right)\left\langle\delta f_{1}^{2}\right\rangle_{\mathscr{H}_{0}}\right) d \boldsymbol{q} d \boldsymbol{p},
\end{aligned}
$$

where $R_{1}=c^{\prime}\left(f_{0}\right)$ and $R_{2}=c^{\prime \prime}\left(f_{0}\right) / 2$. It is quite difficult to obtain explicitly $\delta f_{2}$ satisfying this equation for any $c$ unlike the linear regime. Then, the error $O\left(h^{2}\right)$ is unavoidable in both naive perturbation and T-liearization methods.

In the present article, the form of perturbation is restricted so as to subject to the constraint conditions coming from Casimir invariants at the linear order. By use of the form of constraint conditions (14), it is possible to take into account on the Casimir constraints when we derive the formal stability criterion without use of angle-action variables and this is a topic of forthcoming paper [32].

In this article, we exert the uniform external force to the systems without integrability. We may also consider the case that unperturbed system is integrable but an external force breaks its integrability. It might be an interesting future work, how the local chaos induced by the static external field affects meso- or macro-scopic properties of systems. Such a phenomenon is found in a toy-model with one charged particle confined in cylindrical or toroidal magnetic fields $[33,34]$.

\section{ACKNOWLEDGMENTS}

The author is grateful to Yoshiyuki Y. Yamaguchi and Xavier Leoncini for valuable discussions. He acknowledges the financial support of the A*MIDEX project ( ${ }^{\circ}$ ANR-11IDEX-0001-02) funded by the "investissements d'Avenir" French Government program, managed by the French National Research Agency (ANR).

\section{Appendix A: Conservation of the Casimir functionals (8)}

It is shown that the Casimir functional (8) is conserved in the Vlasov dynamics. Taking the time derivative of $\mathscr{C}[f]$, we have

$$
\frac{d \mathscr{C}[f]}{d t}=\iint \frac{\partial f}{\partial t} c^{\prime}(f) d \boldsymbol{q} d \boldsymbol{p}=-\iint\{\mathscr{H}[f], f\} c^{\prime}(f) d \boldsymbol{q} d \boldsymbol{p} .
$$

Under the conditions asserted above Eq. (8), the boundary terms vanish and the left hand side of Eq. (A1) is

$$
-\iint\{\mathscr{H}[f], f\} c^{\prime}(f) d \boldsymbol{q} d \boldsymbol{p}=\iint \mathscr{H}[f]\left\{f, c^{\prime}(f)\right\} d \boldsymbol{q} d \boldsymbol{p}=0
$$

because $\left\{f, c^{\prime}(f)\right\}=0$. It is then shown that $d \mathscr{C}[f] / d t=0$.

\section{Appendix B: Derivation of Eq. (43)}

We derive Eq. (43). The all we have to do is to perform integration with respect to $\boldsymbol{p}$ in the left hand side of Eq. (43);

$$
\begin{aligned}
& \int_{\mathbf{R}^{2}} \delta(\mathscr{H}(\boldsymbol{q}, \boldsymbol{p})-E) d \boldsymbol{p}=\int_{-\pi}^{\pi} d \theta_{p} \int_{0}^{\infty} p \delta(\mathscr{H}(\boldsymbol{q}, \boldsymbol{p})-E) d p \\
& =2 \pi \int_{0}^{\infty} p \delta\left(\frac{p^{2}}{2}+\mathcal{V}(x, y)-E\right) d p=2 \pi \Theta(E-\mathcal{V}(x, y)),
\end{aligned}
$$

where $\Theta(x)=0$ (resp.1) when $x<0$ (resp. $x \geq 0$ ) is the Heaviside step function, and we have used the relation,

$$
\delta(f(x))=\sum_{x^{*}=f^{-1}(0)} \frac{\delta\left(x-x^{*}\right)}{\left|f^{\prime}\left(x^{*}\right)\right|}, \quad f^{\prime}\left(x^{*}\right) \neq 0 .
$$

Thus we have

$$
\langle g(\boldsymbol{q})\rangle_{E}=\frac{\int_{(-\pi, \pi]^{2}} g(x, y) \Theta(E-\mathcal{V}(x, y)) d x d y}{\int_{(-\pi, \pi]^{2}} \Theta(E-\mathcal{V}(x, y)) d x d y} .
$$

Appendix C: Computation of $\langle\cos x\rangle_{\mathscr{H}_{0}}$ and $\langle\cos x \cos y\rangle_{\mathscr{H}_{0}}$

On the iso- $\mathscr{H}_{0}$ curve, $x$ and $y$ satisfy

$$
\cos x=-\frac{\mathscr{H}_{0}+M \cos y}{M+P \cos y}, \quad \cos y=-\frac{\mathscr{H}_{0}+M \cos x}{M+P \cos x} .
$$

Thus

$$
\begin{aligned}
& \int_{(-\pi, \pi]^{2}} g(x, y) \Theta\left(\mathscr{H}_{0}-\mathcal{V}(x, y)\right) d x d y \\
& =4 \int_{0}^{\arccos \left(-\frac{\mathscr{H}_{0}+M}{M+P}\right)} d x \int_{0}^{\arccos \left(-\frac{\mathscr{H}_{0}+M \cos x}{M+P \cos x}\right)} g(x, y) d y \\
& =4 \int_{0}^{\arccos \left(-\frac{\mathscr{H}_{0}+M}{M+P}\right)} d y \int_{0}^{\arccos \left(-\frac{\mathscr{H}_{0}+M \cos y}{M+P \cos y}\right)} g(x, y) d x
\end{aligned}
$$

for $\mathscr{H}_{0} \in[-2 M-P, P]$ and

$$
\begin{aligned}
& \int_{(-\pi, \pi]^{2}} g(x, y) \Theta\left(\mathscr{H}_{0}-\mathcal{V}(x, y)\right) d x d y \\
& =4 \int_{0}^{\arccos \left(-\frac{\mathscr{H}_{0}-M}{M-P}\right)} d x \int_{0}^{\pi} g(x, y) d y \\
& \quad+4 \int_{\arccos \left(-\frac{\mathscr{H}_{0}-M}{M-P}\right)}^{\pi} d x \int_{0}^{\arccos \left(-\frac{\mathscr{H}_{0}+M \cos x}{M+P \cos x}\right)} g(x, y) d y \\
& =4 \int_{0}^{\arccos \left(-\frac{\mathscr{H}_{0}-M}{M-P}\right)} d y \int_{0}^{\pi} g(x, y) d x \\
& \quad+4 \int_{\arccos \left(-\frac{\mathscr{H}_{0}-M}{M-P}\right)}^{\pi} \int_{0}^{\arccos \left(-\frac{\not \mathcal{H}_{0}+M \cos y}{M+P \cos y}\right)} g(x, y) d x,
\end{aligned}
$$

for $\mathscr{H}_{0} \in[P, 2 M-P]$.

Let us compute $\langle\cos x\rangle_{\mathscr{H}_{0}}=\langle\cos y\rangle_{\mathscr{H}_{0}}$ and $\langle\cos x \cos y\rangle_{\mathscr{H}_{0}}$ 
as follows respectively: When $-2 M-P<\mathscr{H}_{0}<P$, we have

$$
\begin{aligned}
& \langle\cos x\rangle_{\mathscr{H}_{0}}=\langle\cos y\rangle_{\mathscr{H}_{0}} \\
& =\frac{8 \pi}{\sigma\left(\mathscr{H}_{0}\right)} \int_{0}^{\arccos \left(-\frac{\mathscr{H}_{0}+M}{M+P}\right)} \sqrt{1-\left(\frac{\mathscr{H}_{0}+M \cos x}{M+P \cos x}\right)^{2}} d x
\end{aligned}
$$

$\langle\cos x \cos y\rangle_{\mathscr{H}_{0}}=$

$$
\frac{8 \pi}{\sigma\left(\mathscr{H}_{0}\right)} \int_{0}^{\arccos \left(-\frac{\mathscr{H}_{0}+M}{M+P}\right)} \cos x \sqrt{1-\left(\frac{\mathscr{H}_{0}+M \cos x}{M+P \cos x}\right)^{2}} d x
$$

where

$$
\sigma\left(\mathscr{H}_{0}\right)=8 \pi \int_{0}^{\arccos \left(-\frac{\mathscr{H}_{0}+M}{M+P}\right)} \arccos \left(-\frac{\mathscr{H}_{0}+M \cos x}{M+P \cos x}\right) d x,
$$

and where a range of the arccosine function is $[0, \pi]$. When $P<\mathscr{H}_{0}<2 M-P$, we have

$$
\begin{aligned}
& \langle\cos x\rangle_{\mathscr{H}_{0}}=\langle\cos y\rangle_{\mathscr{H}_{0}} \\
& =\frac{8 \pi}{\sigma\left(\mathscr{H}_{0}\right)} \int_{\arccos \left(-\frac{\mathscr{H}_{0}-M}{M-P}\right)}^{\pi} \sqrt{1-\left(\frac{\mathscr{H}_{0}+M \cos x}{M+P \cos x}\right)^{2}} d x,
\end{aligned}
$$

$\langle\cos x \cos y\rangle_{\mathscr{H}_{0}}=$

$$
\frac{8 \pi}{\sigma\left(\mathscr{C}_{0}\right)} \int_{\arccos \left(-\frac{\mathscr{O}_{0}-M}{M-P}\right)}^{\pi} \cos x \sqrt{1-\left(\frac{\mathscr{H}_{0}+M \cos x}{M+P \cos x}\right)^{2}} d x,
$$

where

$$
\begin{aligned}
\sigma\left(\mathscr{H}_{0}\right)= & 8 \pi \int_{\arccos \left(-\frac{\mathscr{H}_{0}-M}{M-P}\right)}^{\pi} \arccos \left(-\frac{\mathscr{H}_{0}+M \cos x}{M+P \cos x}\right) d x \\
& +8 \pi^{2} \arccos \left(-\frac{\mathscr{C}_{0}-M}{M-P}\right) .
\end{aligned}
$$

\section{Appendix D: Integral in $G_{n}(n=1,2,3,4)$}

The integral $\iint F_{0}^{\prime}\left(\mathscr{H}_{0}\right)\langle\cos x\rangle_{\mathscr{H}_{0}}^{2} d \boldsymbol{q} d \boldsymbol{p}$ included in $G_{1}$ and $G_{2}$ is computed as follows;

$$
\begin{aligned}
& \iint F_{0}^{\prime}\left(\mathscr{H}_{0}\right)\langle\cos x\rangle_{\mathscr{H}_{0}}^{2} d \boldsymbol{q} d \boldsymbol{p}=2 \pi \int p d p \int F_{0}^{\prime}\left(\mathscr{H}_{0}\right)\langle\cos x\rangle_{\mathscr{H}_{0}}^{2} d \boldsymbol{q} \\
& =2 \pi \int_{-2 M-P}^{2 M-P} d \mathscr{H}_{0} F_{0}^{\prime}\left(\mathscr{H}_{0}\right)\langle\cos x\rangle_{\mathscr{H}_{0}}^{2} \int \Theta\left(\mathscr{H}_{0}+\mathscr{V}(x, y)\right) d \boldsymbol{q} \\
& =2 \pi \int_{-2 M-P}^{2 M-P} F_{0}^{\prime}\left(\mathscr{H}_{0}\right)\langle\cos x\rangle_{\mathscr{H}_{0}}^{2} \sigma\left(\mathscr{H}_{0}\right) d \mathscr{H}_{0},
\end{aligned}
$$

where $p=\|\boldsymbol{p}\|=\sqrt{2\left(\mathscr{H}_{0}+\mathcal{V}(x, y)\right)}$ and $\sigma\left(\mathscr{H}_{0}\right)$ is defined in Eqs. (C6) and (C9). The similar terms in $G_{2}$ and $G_{4}$ are computed in the same manner.
[1] A. Campa, T. Dauxois, D. Fanelli, and S. Ruffo, Physics of LongRange Interacting Systems, (Oxford University Press, Oxford, 2014).

[2] A. Campa, T. Dauxois, and S. Ruffo, Statistical mechanics and dynamics of solvable models with long-range interactions, Phys. Rep. 480, 57 (2009).

[3] J. Binney and S. Tremaine, Galactic dynamics, 2nd ed. (Princeton University Press, Princeton, NJ, 2008).

[4] Y. Y. Yamaguchi, J. Barré, F. Bouchet, T. Dauxois, and S. Ruffo, Stability criteria of the Vlasov equation and quasi-stationary states of the HMF model, Physica A 337, 36 (2004).

[5] J. Barré, F. Bouchet, T. Dauxois, S. Ruffo, and Y. Y. Yamaguchi, The Vlasov equation and the Hamiltonian mean-field model, Physica A 365, 177 (2006).

[6] W. Braun and K. Hepp, The Vlasov dynamics and its fluctuations in the $1 / N$ limit of interacting classical particles, Commun. Math. Phys. 56, 101 (1977).

[7] R. L. Dobrushin, Vlasov equations, Funct. Anal. Appl. 13, 115 (1979).

[8] H. Spohn, Large scale dynamics of interacting particles, (Springer-Verlag, Heidelberg, 1991).

[9] A. Boozer, Physics of magnetically confined plasmas, Rev. Mod. Phys. 76, 1071 (2005).

[10] A. Patelli, S. Gupta, C. Nardini, and S. Ruffo, Linear response theory for long-range interacting systems in quasistationary states, Phys. Rev. E 85, 021133 (2012).
[11] S. Ogawa and Y. Y. Yamaguchi, Linear response theory in the Vlasov equation for homogeneous and for inhomogeneous quasistationary states, Phys. Rev. E 85, 061115 (2012).

[12] P. H. Chavanis, Linear response theory for hydrodynamic and kinetic equations with long-range interactions, Eur. Phys. J. Plus 128, 38 (2013).

[13] S. Ogawa, A. Patelli, and Y. Y. Yamaguchi, Non-mean-field critical exponent in a mean field model: Dynamics versus statistical mechanics, Phys. Rev. E 89, 032131 (2014).

[14] S. Ogawa and Y. Y. Yamaguchi, Nonlinear response for external field and perturbation in the Vlasov system, Phys. Rev. E 89, 052114 (2014).

[15] S. Ogawa and Y. Y. Yamaguchi, Landau-like theory for universality of critical exponents in quasistationary states of isolated mean-field systems Phys. Rev. E 91, 062108 (2015).

[16] A. Patelli and S. Ruffo, General linear response formula for non integrable systems obeying the Vlasov equation, Eur. Phys. J. D 68, 329 (2014).

[17] S. Ogawa, Spectral and formal stability criteria of spatially inhomogeneous stationary solutions to the Vlasov equation for the Hamiltonian mean-field model, Phys. Rev. E 87, 062107 (2013).

[18] A. Campa and P. H. Chavanis, A dynamical stability criterion for inhomogeneous quasi-stationary states in long-range systems J. Stat. Mech, P06001 (2010). 
[19] C. Lancellotti and J. J. Dorning, Critical Initial States in Collisionless Plasmas, Phys. Rev. Lett. 81, 5137 (1998).

[20] C. Lancellotti and J. J. Dorning, Time-asymptotic wave propagation in collisionless plasmas, Phys. Rev. E 68, 026406 (2003).

[21] C. Lancellotti and J. J. Dorning, Nonlinear Landau damping, Transp. Theory Stat. Phys. 38, 1 (2009).

[22] Y. Y. Yamaguchi and S. Ogawa, Conditions for predicting quasistationary states by rearrangement formula, Phys. Rev. E 92, 042131 (2015).

[23] A. C. Ribeiro-Teixeira, F. P. C. Benetti, R. Pakter, and Y. Levin, Ergodicity breaking and quasistationary states in systems with long-range interactions. Phys. Rev. E 89, 022130 (2014).

[24] F. P. C. Benetti, A. C. Ribeiro-Teixeira, R. Pakter, and Y. Levin, Nonequilibrium Stationary States of 3D Self-Gravitating Systems, Phys. Rev. Lett. 113, 100602 (2014).

[25] D. D. Holm, J. E. Marsden, T. Ratiu, and A. Weinstein, Nonlinear stability of fluid and plasma equilibria, Phys. Rep. 123, 1 (1985).

[26] J. Barré, A. Olivetti, and Y. Y. Yamaguchi, Algebraic damping in the one-dimensional Vlasov equation, J. Phys. A: Math. Theor. 44405502 (2011).

[27] J. Barré and Y. Y. Yamaguchi, On algebraic damping close to inhomogeneous Vlasov equilibria in multi-dimensional spaces, J. Phys. A: Math. Theor. 46, 225501 (2013).

[28] M. Antoni and A. Torcini, Anomalous diffusion as a signature of a collapsing phase in two-dimensional self-gravitating systems, Phys. Rev. E 57, R6233 (1998).

[29] A. Torcini and M. Antoni, Equilibrium and dynamical properties of two-dimensional $N$-body systems with long-range attractive interactions, Phys. Rev. E 59, 2746 (1999).

[30] H. Yoshida, Recent progress in the theory and application of symplectic integrators, Celest. Mech. Dynam. Astron. 56, 27 (1993).

[31] F. Bouchet and H. Morita, Large time behavior and asymptotic stability of the 2D Euler and linearized Euler equations, Physica D 239, 948 (2010).

[32] S. Ogawa, Stability criterion of spatially inhomogeneous solutions to Vlasov equation, submitted.

[33] B. Cambon, X. Leoncini, M. Vittot, R. Dumont, and X. Garbet, Chaotic motion of charged particles in toroidal magnetic configurations, Chaos: An Interdisciplinary Journal of Nonlinear Science 24, 033101 (2014).

[34] S. Ogawa, B. Cambon, X. Leoncini, M. Vittot, D. del CastilloNegrete, G. Dif- Pradalier, and X. Garbet, Full particle orbit effects in regular and stochastic magnetic fields, Phys. Plasmas 23, 072506 (2016). 